\title{
THE ADDITIVE GENETIC VARIANCE AFTER BOTTLENECKS IS AFFECTED BY THE NUMBER OF LOCI INVOLVED IN EPISTATIC INTERACTIONS
}

\author{
YAMAMA NACIRI-GRAVEN ${ }^{1}$ AND JÉRÔME GOUdET ${ }^{2}$ \\ Institute of Ecology, Biology Building, Lausanne University, CH 1015 Lausanne, Switzerland \\ ${ }^{2}$ E-mail: Jerome.Goudet@ie-zea.unil.ch
}

\begin{abstract}
We investigated the role of the number of loci coding for a neutral trait on the release of additive variance for this trait after population bottlenecks. Different bottleneck sizes and durations were tested for various matrices of genotypic values, with initial conditions covering the allele frequency space. We used three different types of matrices. First, we extended Cheverud and Routman's model by defining matrices of "pure" epistasis for three and four independent loci; second, we used genotypic values drawn randomly from uniform, normal, and exponential distributions; and third we used two models of simple metabolic pathways leading to physiological epistasis. For all these matrices of genotypic values except the dominant metabolic pathway, we find that, as the number of loci increases from two to three and four, an increase in the release of additive variance is occurring. The amount of additive variance released for a given set of genotypic values is a function of the inbreeding coefficient, independently of the size and duration of the bottleneck. The level of inbreeding necessary to achieve maximum release in additive variance increases with the number of loci. We find that additive-by-additive epistasis is the type of epistasis most easily converted into additive variance. For a wide range of models, our results show that epistasis, rather than dominance, plays a significant role in the increase of additive variance following bottlenecks.
\end{abstract}

Key words.-Additive genetic variance, epistasis, inbreeding coefficient, multiple loci, population bottlenecks.

Received July 23, 2002. Accepted November 25, 2002.

The role of population bottlenecks in reducing the genetic variability has often been emphasized because reduced variability is thought to alter the evolutionary potential of populations (Nei et al. 1975; Falconer and Mackay 1996). In purely additive models of quantitative variation, the loss of genetic variation resulting from a population bottleneck is expected to decrease the amount of additive variance $\left(\sigma_{A}^{2}\right)$, and thus the capacity of bottlenecked populations to respond to selection (Wright 1931; Lande 1980). But Wright (1931) also put forward the idea that evolution could be favored in small populations. In the first phase of his Shifting Balance Theory of Evolution, Wright proposed that allele frequencies at multiple loci fluctuate as a consequence of genetic drift and therefore explore the allelic frequency space, allowing new favorable gene combinations to appear. Fisher (1958) strongly opposed to this view, dismissed the importance of drift for evolution and focused his attention on individual effects of genes on fitness in large populations.

Experimental and theoretical studies have since demonstrated that additive genetic variance (and therefore an enhanced ability to evolve) can increase after episodes of reduced population sizes. Experimentally, the phenomenon has been observed for morphometric traits as well as behavioral traits in the housefly Musca domestica (for a review, see Meffert 2000), and for components of fitness in Drosophila melanogaster (López-Fanjul and Villaverde 1989; Garcia et al. 1994), Tribolium castaneum (Fernández et al. 1995; Wade et al. 1996), Bicyclus anynana (Saccheri et al. 1996), and Mus musculus (Cheverud et al. 1999). Theoretical studies by Robertson (1952), Willis and Orr (1993), and Wang et al. (1998) have shown that dominance can explain

\footnotetext{
${ }^{1}$ Present address: Laboratoire de Systématique et de Génétique Moléculaires. Conservatoire et Jardin Botaniques. Chemin de l'Impératrice, 1. CH 1292 Chambésy, Geneva. Switzerland; E-mail: Yamama.Naciri-Graven@cjb.ville-ge.ch.
}

an increase in additive variance following a bottleneck, whereas Goodnight (1987, 1988); Cockerham and Tashida (1988); Whitlock et al. (1993) as well as Cheverud and Routman $(1995,1996)$ have suggested that the loss of additive variance following a bottleneck could be limited or even reversed with epistasis. Accordingly, some of the experimental studies have emphasized the role played by inbreeding and dominance in inflating the additive variance after population bottlenecks (López-Fanjul and Villaverde 1989; Garcia et al. 1994; Fernández et al. 1995; Saccheri et al. 1996) whereas others have found their results to be a confirmation that epistasis can be converted into additive variance (Bryant and Meffert 1995, 1996; Cheverud et al. 1999). Although models of dominance have been extensively studied in the past years at a theoretical level, through studies on the effect of dominance on additive variance (Robertson 1952; Rose 1982; Willis and Orr 1993; Wang et al. 1998; López-Fanjul et al. 2002) or mutation accumulation experiments (Lynch et al. 1995; Keightley and Eyre-Walker 1999), models of epistatic interactions have been less explored and were based on only two interacting loci (Goodnight 1987, 1988; Cheverud and Routman 1995, 1996; López-Fanjul et al. 1999, 2000, 2002; see also the recent book edited by Wolf et al. 2000). However, the number of loci underlying quantitative traits is usually larger than two (Lynch and Walsh 1998) and this could be all the more true for fitness traits (Merilä and Sheldon 1999).

In this paper we investigate how epistatic interactions between up to four biallelic independent loci coding for a neutral trait can influence the amount of additive variance generated after population bottlenecks of varying sizes. Our aim is to test the hypothesis that the number of genes (two, three, or four) involved in interactions is an important factor affecting the release in additive variance after population bottlenecks, using different matrices of genotypic values. 


\section{THEORY}

Our study builds on the Markov Chain transition of allele frequencies of a Wright-Fisher model with an initial infinite population from which bottlenecked populations are derived. We simulated four independent neutral biallelic loci, coding for a neutral trait.

\section{Genetic Effects for the Four Locus Model}

Calling $G_{i j k l m n o p}$ the genotypic value of genotype $i j$ at locus $1, k l$ at locus $2, m n$ at locus 3 , and op at locus 4, additive and dominance effects were computed for the four locus model using the general least squares model for genetic effects (Lynch and Walsh 1998, pp. 84-85) as follows:

$$
\begin{aligned}
\alpha_{i} & =G_{i \ldots \ldots}-\mu_{G} \\
\beta_{i j} & =G_{i j \ldots \ldots}-\mu_{G}-\alpha_{i}-\alpha_{j}
\end{aligned}
$$

where $\alpha_{i}$ is the additive effect of allele $i, \beta_{i j}$ is the dominance effect between alleles $i$ and $j, \mu_{G}$ is the population mean genotypic value, and $G_{i \ldots \ldots}$ (respectively, $G_{i j \ldots . . .}$ ) the conditional mean genotypic value of individuals with allele $i$ (respectively with alleles $i$ and $j$ ) at the first locus. We restricted the study to the case of biallelism at each locus $(i, j, k, l, m$, $n, o, p \in[1,2])$. Similar definitions hold for the second $\left(\alpha_{k}\right.$, $\left.\beta_{k l}\right)$, the third $\left(\alpha_{m}, \beta_{m n}\right)$, and the fourth gene $\left(\alpha_{o}, \beta_{o p}\right)$. The additive $\left(\sigma_{A}^{2}\right)$ and dominance $\left(\sigma_{D}^{2}\right)$ variances were then derived as:

$$
\sigma_{A}^{2}=\sum_{i=1}^{4} \sum_{j=1}^{2} 2 p_{i j} \alpha_{i j}^{2}
$$

where $i$ indices the locus and $j$ the allele,

$$
\sigma_{D}^{2}=\sum_{i=1}^{4} \sum_{j=1}^{2} \sum_{k=1}^{2} p_{i j k} \beta_{i j k}^{2}
$$

where $i$ indexes loci and $j$ and $k$ the two alleles at the $i$ locus.

The epistatic variance $\left(\sigma_{I}^{2}\right)$ was computed as the difference between the genetic variance and its additive and dominance components:

$$
\sigma_{I}^{2}=\sigma_{G}^{2}-\left(\sigma_{A}^{2}+\sigma_{D}^{2}\right)
$$

where $\sigma_{G}^{2}$ is the genetic variance of the population defined as:

$$
\sigma_{G}^{2}=\sum_{i, j, k, l, m, n, o, p} p_{i j k l m n o p} G_{i j k l m n o p}^{2}-\mu_{G}^{2}
$$

This mode of calculation for the interaction variance implies that we cannot separate its underlying components, in terms of additive-by-additive (AA), additive-by-dominance (AD) and dominance-by-dominance (DD) types of epistasis (see below). But this separation would have been cumbersome, since each component is represented by terms for two, three, and four loci interactions.

For the two locus model, one allele was fixed at the first and second loci, whereas it was fixed at the first locus only for the three locus model.

\section{Numerical Computations}

We ran numerical computations based on the Markov chain transition of allele frequency in a Wright-Fisher model de- scribed in Hartl and Clark (1997). In a population of constant size $N$, allele copy number can vary between 0 and $2 N$. Under the effect of drift only, the probability of moving from $a$ copies in generation $t$ to $b$ copies in generation $(t+1)$ is $T_{a b}$ $=\left(b^{2 N}\right)(a / 2 N)^{b}(1-a / 2 N)^{2 N-b}$. These coefficients were stored in a matrix $([2 N+1] \times[2 N+1])$ for all the generations after the first bottleneck and up to the twentieth generation. For the transition between the initial infinite population and the first bottlenecked generation, we consider 11 allele frequencies, from 0 to 1 by steps of 0.1 . The transition matrix from the initial to the first generation was thus $(11 \times[2 N+$ 1]). Bottleneck sizes were set at $N=2$, 4, or 8 individuals and the inbreeding coefficient in the randomly mating bottlenecked populations was estimated as $F=1-(1-1 / 2 N)^{\mathrm{t}}$. This inbreeding coefficient was used as a scale on all the figures, since it combines adequately bottleneck size and number of generations (it should be kept in mind that this inbreeding coefficient is the direct result of drift in allele frequencies, rather than increased homozygosity at constant allele frequencies). The matrices of genotypic values used in the simulations are described below. Each simulation was run for twenty generations. The average magnitude of the change in additive variance following a bottleneck was quantified by looking at the standardized release in additive variance $\left(R_{\left(t_{i}-t_{0}\right)}\right)$ between the initial infinite population and the $i^{\text {th }}$ bottlenecked generation derived from it. $R_{\left(t_{i}-t_{0}\right)}$ is estimated over all the allele frequency space as:

$$
R_{\left(t_{i}-t_{0}\right)}=\frac{1}{11^{4}} \sum_{p 1} \sum_{p 2} \sum_{p 3} \sum_{p 4}\left(\sigma_{A i}^{2}-\sigma_{A 0}^{2}\right) / \sigma_{G 0}^{2},
$$

where $\sigma_{A 0}^{2}$ and $\sigma_{A i}^{2}$ are the additive variances in the initial infinite population and after $i$ generations of bottleneck respectively, $\sigma_{G 0}^{2}$ is the initial total genetic variance and $p 1$, $p 2, p 3$, and $p 4$ index allele frequencies at locus $1,2,3$, and 4 , respectively. $R_{\left(t_{i}-t_{0}\right)}$ was used to estimate both the amount and the direction of the changes in additive variance since $R_{\left(t_{i}-t_{0}\right)}$ might be either positive or negative. An important point to emphasize is that $R_{\left(t_{i}-t_{0}\right)}$ is the proportion of changes in additive variance relative to the initial total genetic variance. It is thus unaffected by the mean and variance of the matrix of genotypic values. Owing to this standardization, our results cannot be considered as an artifact due to changes in the over all level of genetic variance (Keightley 1989). An associated quantity of interest is the maximum amount of release in additive variance achieved. This quantity will be noted Max $R_{\left(t_{i}-t_{0}\right)}$ below. Programs to carry out these calculations are available upon request from JG.

\section{Genotypic Values}

Three different sets of genotypic values were used in the simulations. The first set is a generalization to four loci of "pure" forms of additive-by-additive (AA), additive-bydominance (AD), and dominance-by-dominance (DD) epistasis described in Cheverud and Routman (1996). In these matrices, both $a$ (the single locus additive value defined as half the difference between homozygotes) and $d$ (the single locus dominance value defined as the difference between the heterozygote and the mean of the two homozygotes) were always 0 for a given locus when the effects of the remaining 
TABLE 2. Proportion of the different variance components in the initial infinite population. These proportions are averages over the allele frequency space, with frequencies ranging from 0 to 1 by step of 0.1 . Mean of the ten matrices with standard deviation in parentheses.

\begin{tabular}{|c|c|c|c|c|c|c|c|c|c|}
\hline \multirow{2}{*}{$\begin{array}{c}\text { Variance } \\
\text { components }\end{array}$} & \multirow{2}{*}{$\begin{array}{l}\text { Gene } \\
\text { number }\end{array}$} & \multicolumn{3}{|c|}{ "Pure" epistasis } & \multicolumn{3}{|c|}{ Statistical distributions } & \multicolumn{2}{|c|}{$\begin{array}{l}\text { Physiological } \\
\text { epistasis }\end{array}$} \\
\hline & & AA & $\mathrm{AD}$ & DD & Exponential & Uniform & Normal & DMP & AMP \\
\hline Additivity & $\begin{array}{l}2 \\
3 \\
4\end{array}$ & $\begin{array}{l}70 \\
45 \\
27\end{array}$ & $\begin{array}{c}33 \\
22-11^{\mathrm{a}} \\
7\end{array}$ & $\begin{array}{r}15 \\
5 \\
2\end{array}$ & $\begin{array}{l}41.8(9.0) \\
30.3(4.4) \\
20.5(2.2)\end{array}$ & $\begin{array}{l}35.0(13.7) \\
28.1(6.8) \\
21.4(2.2)\end{array}$ & $\begin{array}{l}46.5(13.1) \\
30.0(7.7) \\
22.4(2.5)\end{array}$ & $\begin{array}{l}63 \\
56 \\
48\end{array}$ & $\begin{array}{l}84 \\
67 \\
52\end{array}$ \\
\hline Dominance & $\begin{array}{l}2 \\
3 \\
4\end{array}$ & $\begin{array}{l}0 \\
0 \\
0\end{array}$ & $\begin{array}{c}25 \\
12-12^{\mathrm{a}} \\
6\end{array}$ & $\begin{array}{r}25 \\
8 \\
3\end{array}$ & $\begin{array}{l}36.3(6.1) \\
25.3(5.7) \\
16.3(2.4)\end{array}$ & $\begin{array}{l}34.9(10.6) \\
26.7(6.7) \\
17.2(2.5)\end{array}$ & $\begin{array}{l}26.2(9.9) \\
23.0(6.0) \\
16.5(2.8)\end{array}$ & $\begin{array}{l}26 \\
19 \\
14\end{array}$ & $\begin{array}{l}0 \\
0 \\
0\end{array}$ \\
\hline
\end{tabular}

a The two figures are for ADAD and DADA genotypic matrices.

loci were summed. In our generalization, we insured that $a$ and $d$ remains equal to zero over all loci, while allowing for epistatic interactions of higher order to be present. For instance, in the AAAA matrix "pure" AA, AAA, and AAAA epistatic interactions are present. To account for the asymmetry of the three locus model, four matrices were designed, corresponding to AAAA, ADAD, DADA, and DDDD epistasis (see Appendix).

Next, genotypic values were chosen at random from three distributions. First we used the exponential distribution because numerous QTL analyses show a L-shaped distribution of gene effects (Lynch and Walsh 1998; Bost et al. 1999). Moreover, in a QTL-simulation study Otto and Jones (2000) showed that there is a strong theoretical support in using the exponential distribution to fit gene effects distribution. We also used normal and uniform distributions in order to mimic different levels of variance, skewness, and kurtosis in the distribution of genotypic values. For each of these distributions, 10 matrices of 81 genotypic values were drawn randomly.

Last, two matrices of genotypic values were built to mimic a simple metabolic pathway consisting of two to four steps (according to the number of loci). As before, the loci were biallelic. For these two matrices, allele 1 is not functional and therefore homozygotes 1:1 at any of the loci have a genotypic value of 0 . In the first matrix, called dominant metabolic pathway (DMP), the presence of 1 copy of allele 2 at each locus is sufficient to restore complete functioning of the pathway (genotypic value of 1). In the second, called additive metabolic pathway (AMP), the heterozygote 1:2 produces half the amount of metabolites produced by homozygote 2:2 at each locus. Therefore, the quadruple homozygote (genotypic value of 16) produces 16 times as much as the quadruple heterozygotes (genotypic value of 1 , see Appendix). According to Templeton (2000) and Goodnight (2000), these forms of epistasis can be referred as physiological, or functional epistasis. It is not so for matrices derived from Cheverud and Routman's model which correspond to "pure" forms of epistasis, without any reference to gene action.

For the second and the third sets of genotypic values, additive and dominance effects exist in addition to epistatic effects, so that changes in additive variance are not due to epistatic conversion only (this is also the case for "pure", forms of epistasis, when the allele frequencies are different from 0.5). All these matrices correspond to the genotypic values of traits that are neutral, and therefore not subject to selection.

\section{RESULTS}

Table 1 clearly shows that for all genotypic matrices the proportion of epistatic variance in the initial infinite population increases with the number of loci, and both additive and dominance variance proportions diminish accordingly. Among the randomly drawn matrices, the apportionment of the different types of variance is very similar and more similar as the number of genes increases (note the low standard deviations in Table 1 for the four locus model). The largest difference in the proportion of the different variances is seen among the matrices of "pure", epistatic forms, in particular between the AA and DD matrices.

The average release in additive variance following a bottleneck, $R_{\left(t_{i}-t_{0}\right)}$, is plotted on Figures 1 to 3 as a function of the inbreeding coefficient.

\section{“Pure”' Epistasis}

The results for " $p u r e$ " epistasis are shown in Figure 1. For two, three, and four loci, it is "pure" AA epistasis that leads to the largest increase in additive variance (panel A). With two genes, the average release in additive variance $\left(R_{\left(t_{i}-t_{0}\right)}\right)$ is never positive except with AA epistasis. With three and four interacting genes, $R_{\left(t_{i}-t_{0}\right)}$ becomes positive for each of the "pure" epistasis types. With AA epistasis and two genes, $R_{\left(t_{i}-t_{0}\right)}$ is maximum $(5 \%)$ for $F=0.23$ and becomes negative when $F$ is larger than 0.43 . With three genes, $R_{\left(t_{i}-t_{0}\right)}$ is maximum (40\%) for $F=0.50$ and becomes negative when $F$ is higher than 0.85 . With four genes, the maximum release of additive variance reaches $88 \%$ for an inbreeding coefficient of 0.62 , and additive variance diminishes compared to the initial population for $F$ larger than 0.96. DD is the type of "pure" epistasis for which the maximum $R_{\left(t_{i}-t_{0}\right)}$ is lowest (panel D), even with the four locus model (Max $R_{\left(t_{i}-t_{0}\right)}=2 \%$ and $F=0.54$ ), whereas AD epistasis (panels B and C) is intermediate (Max $R_{\left(t_{i}-t_{0}\right)}=14 \%$ and $F$ $=0.60$ for the four locus model). 
A

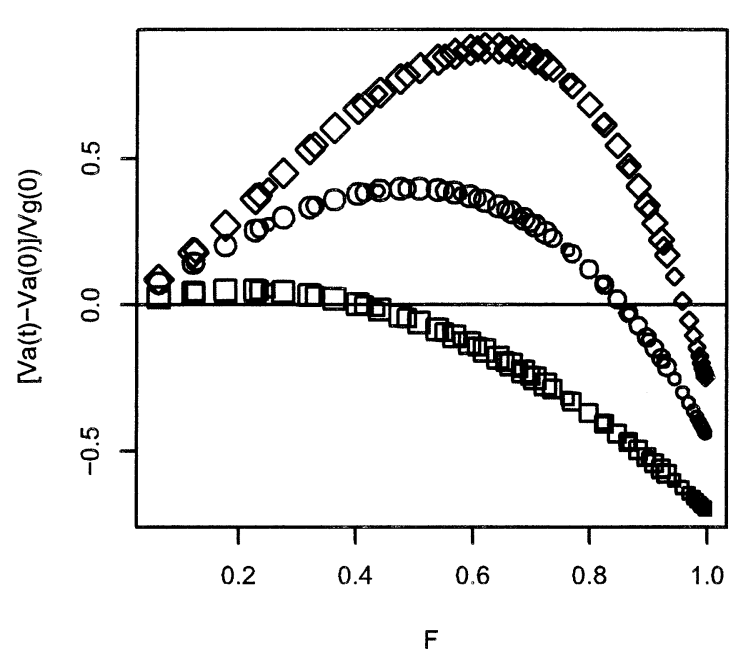

C

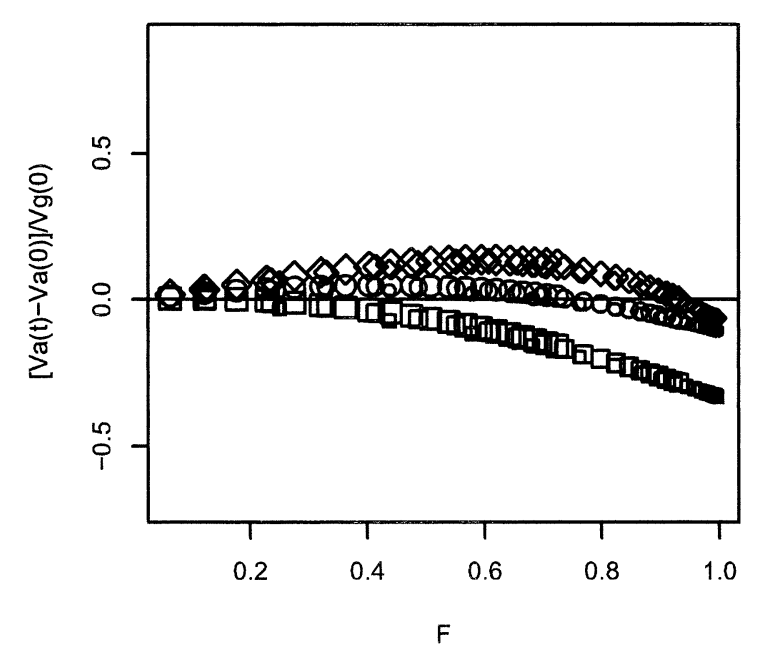

B

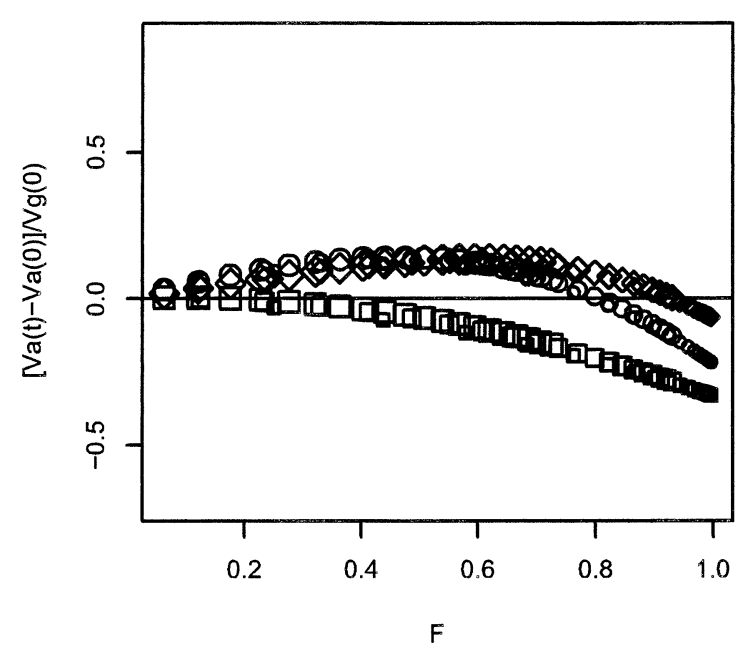

D

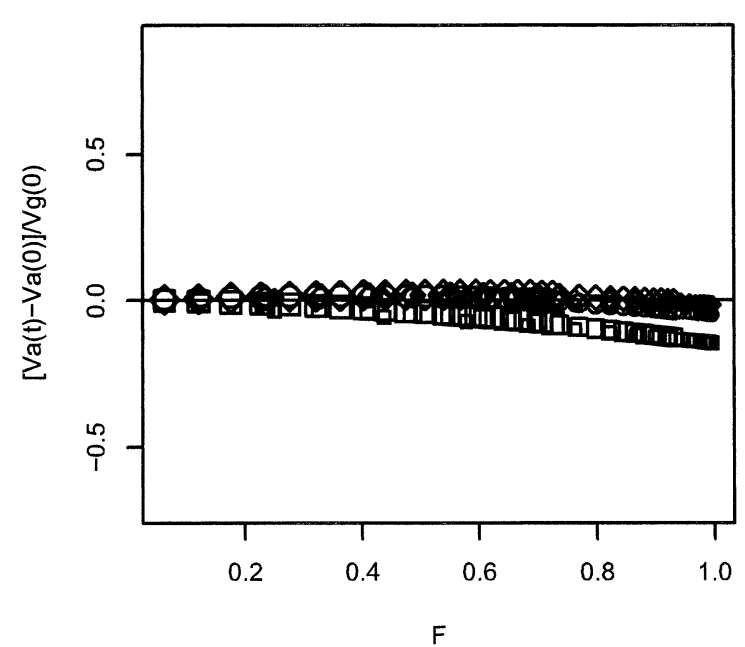

FIG. 1. Average release in additive variance after bottlenecks of size 2, 4, and 8, expressed as the average over the whole frequency space of $R\left(t-t_{0}\right)=\left(\sigma_{A}^{2}[t]-\sigma_{A}^{2}\left[t_{0}\right]\right) / \sigma_{G}^{2}\left(t_{0}\right)$ as a function of the inbreeding coefficient $\left(F=1-(1-1 / 2 N)^{t}\right)$ for "pure" epistasis. $(\mathrm{A})$ Additive-additive epistasis (AA and AAA and AAAA); (B) Additive-dominance epistasis (AD and ADA and ADAD); (C) Dominanceadditive epistasis (DA and DAD and DADA); (D) Dominance-dominance epistasis (DD and DDD and DDDD). Squares: two locus model; circles: three locus model; and diamonds: four locus model. Small sized symbols are for bottleneck of size 2, medium sized symbols are for bottlenecks of size 4 , and large sized symbols are for bottlenecks of size 8.

For the two and four locus models with mixed "pure" epistasis effects ADAD and DADA (panels B and C), the order in which additive and dominance effects are entered makes no difference. For the three locus model, DAD epistasis displays a pattern similar to DDD, whereas ADA epistasis displays a pattern similar to AAA.

\section{Random Matrices of Genotypic Values}

The results for random matrices of genotypic values are shown on Figure 2. For the two locus model, $R_{\left(t_{i}-t_{0}\right)}$ is nearly always negative. $R_{\left(t_{i}-t_{0}\right)}$ becomes positive in the three and four locus models for an important range of inbreeding level $(0<F \leq 0.50$ and $0<F \leq 0.70$, respectively). The max- imum $R_{\left(t_{i}-t_{0}\right)}$ is observed for the four locus model no matter what the underlying distribution, but the exponential matrices (panel A) give on average the higher $R_{\left(t_{i}-t_{0}\right)}$ values, followed by matrices generated from the uniform (panel C) and the normal (panel B). The maximum release in additive variance is below $10 \%$ on average and none of the 30 matrices showed a maximum release in additive variance in excess of $15 \%$. We observed that for four genes, all the 30 matrices showed at least some increase in additive variance following a bottleneck. As is apparent from Figure 2, the differences in the average release of additive variance among the three types of distributions are quantitative rather than qualitative. 
A

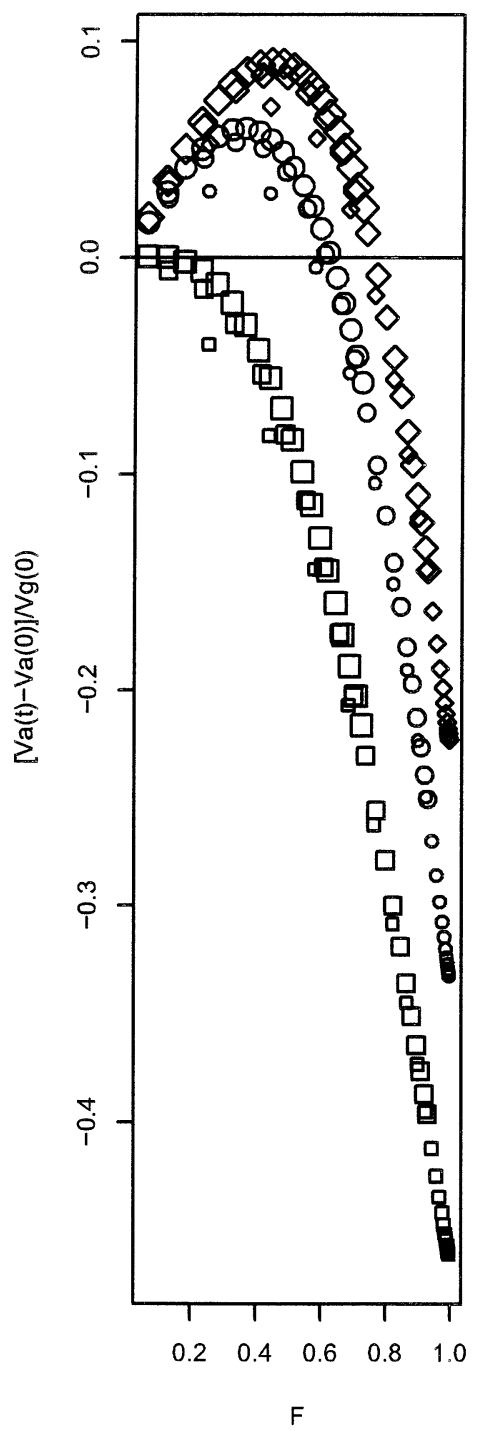

B

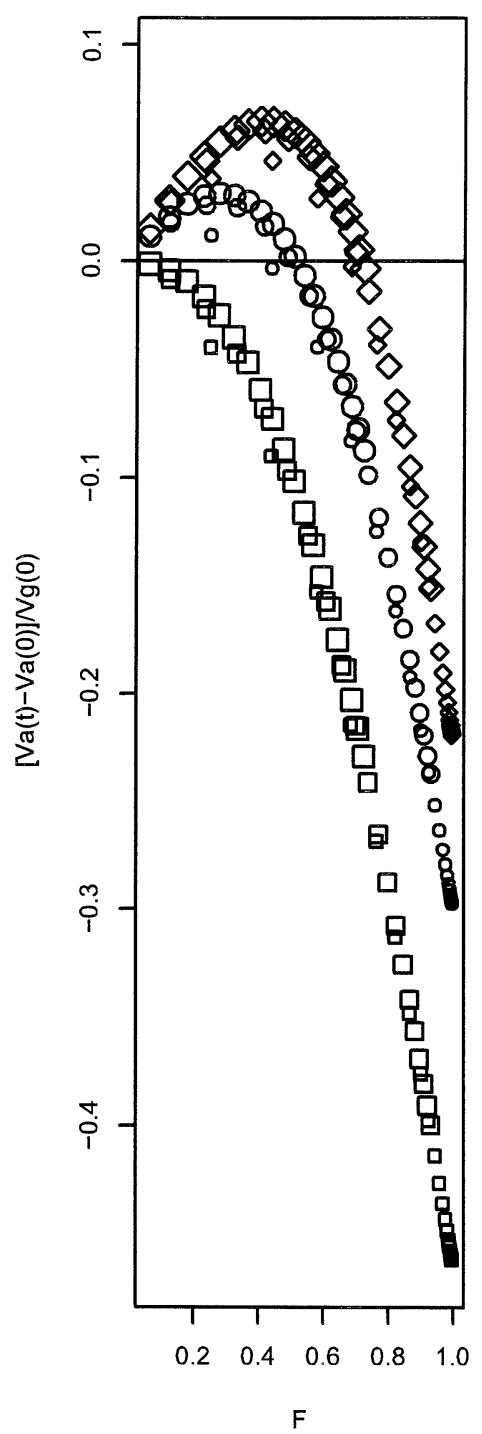

C

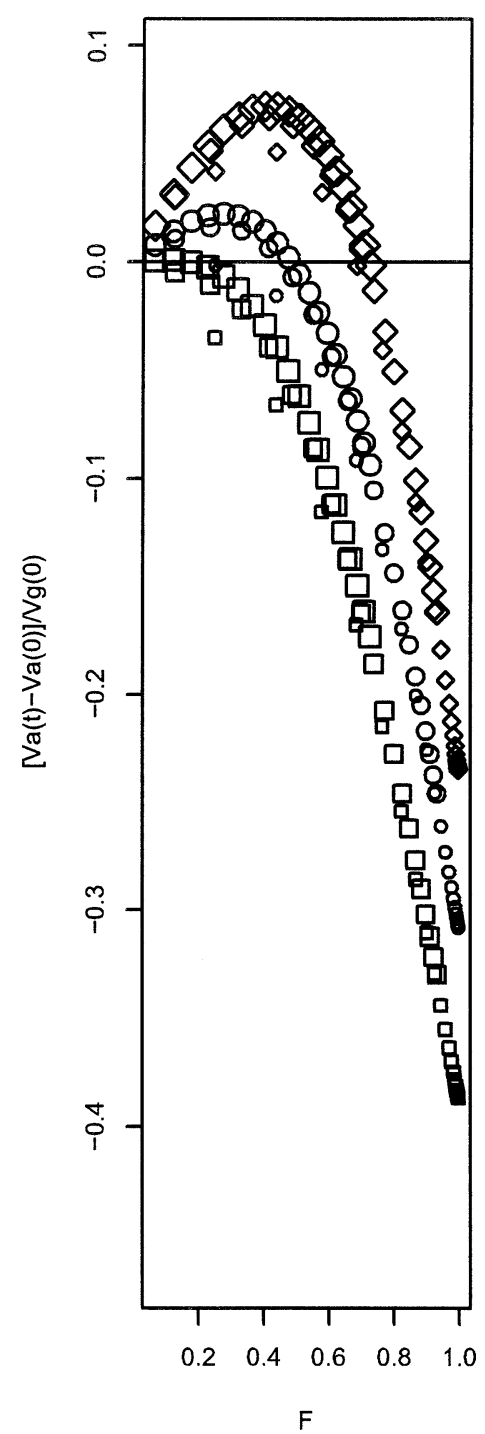

FIG. 2. Average release in additive variance after bottlenecks of size 2, 4, and 8 , expressed as the average over the whole frequency space of $R\left(t-t_{0}\right)=\left(\sigma_{A}^{2}[t]-\sigma_{A}^{2}\left[t_{0}\right]\right) / \sigma_{G}^{2}\left(t_{0}\right)$ as a function of the inbreeding coefficient $(F)$ for exponential (A), normal (B), and uniform (C) distributions. Squares: two locus model; circles: three locus model; and diamonds: four locus model. Small sized symbols are for bottleneck of size 2, medium sized symbols are for bottlenecks of size 4, and large sized symbols are for bottlenecks of size 8 .

\section{Metabolic Pathways}

The two simple metabolic pathways show very different patterns (Fig. 3). When there is dominance at each locus (DMP model, panel A), a bottleneck always reduces the amount of additive variance and the trend is little affected by the number of loci. In striking contrast, when alleles at each locus interact additively (AMP model, panel B), a bottleneck will reduce additive variance when interactions take place among two loci, but additive variance will be increased for three and four loci over a large range of inbreeding values ( $F$ between 0 and 0.5 for three genes and up to 0.8 for four genes). Increases could also be very substantial, as the maximum $R_{\left(t_{i}-t_{0}\right)}$ for four genes is $28 \%$.

From the inspection of these figures, three major trends are noticeable: (1) increasing the number of genes underlying quantitative characters increases the release in additive variance or slows down its loss. (2) For a given genotypic value and number of genes, it is the inbreeding coefficient rather than the size of the bottleneck that determines mostly the release in additive variance. (3) As the number of genes increases, the inbreeding coefficient for which there is a maximum release in additive variance also increases. We also observe that for a given number of loci and over the three different types of genotypic matrices ("pure," physiological, or statistical epistasis), no clear relation emerges between the way genetic variance is initially partitioned into additivity, dominance, and epistasis and the amount of release in additive variance after population bottlenecks (Table 1 and Figs. $1-3)$. 
A

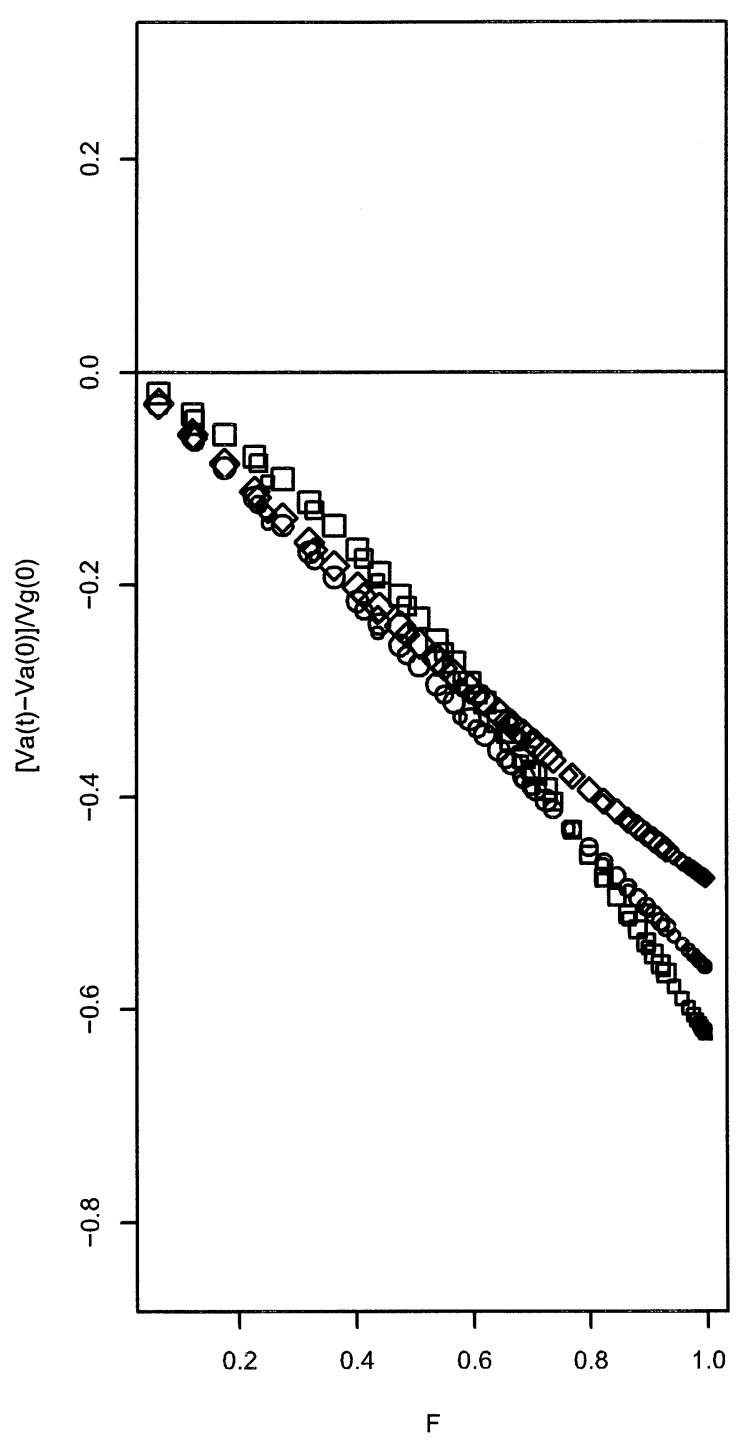

B

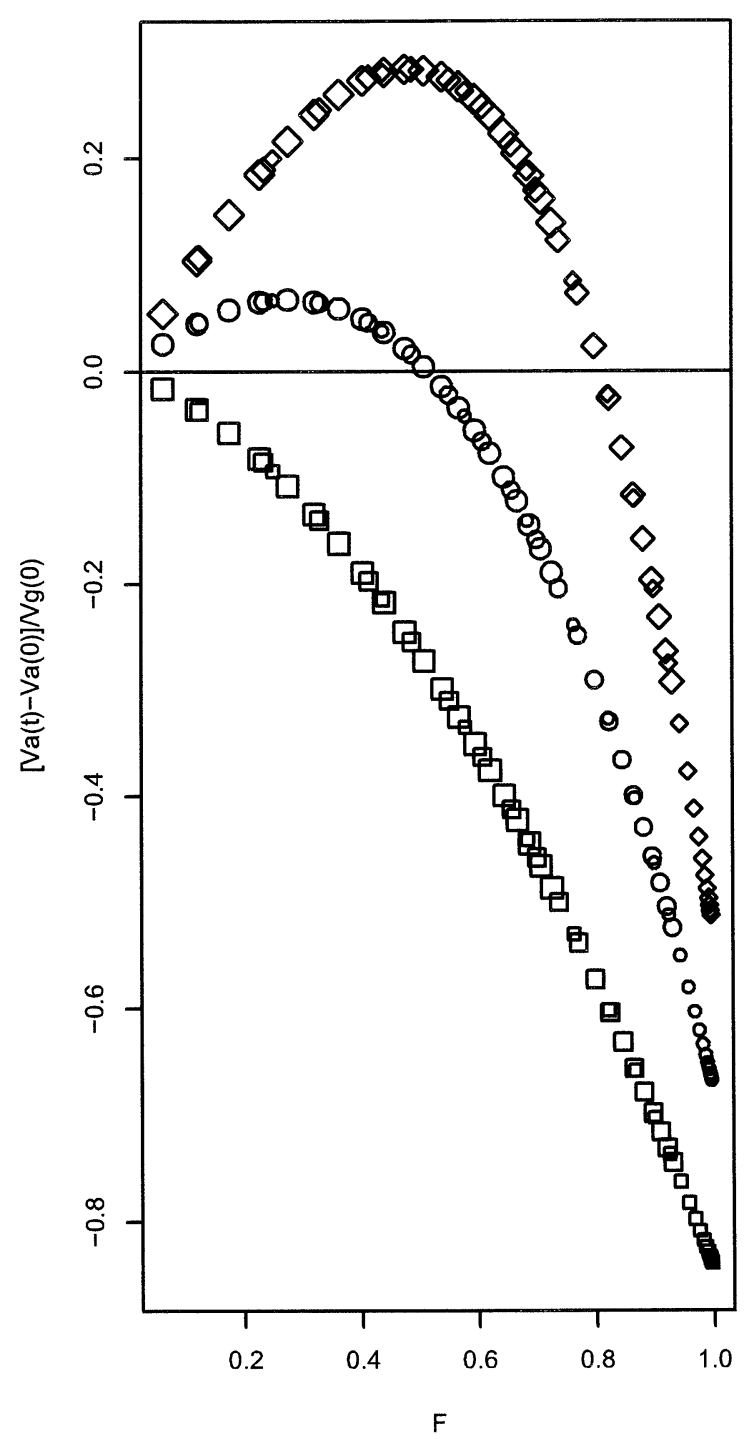

FIG. 3. Average release in additive variance after bottlenecks of size 2, 4, and 8 , expressed as the average over the whole frequency space of $R\left(t-t_{0}\right)=\left(\sigma_{A}^{2}[t]-\sigma_{A}^{2}\left[t_{0}\right]\right) / \sigma_{G}^{2}\left(t_{0}\right)$ as a function of the inbreeding coefficient $(F)$ for the two models of physiological epistasis: dominant metabolic pathway (A) and additive metabolic pathway (B). Squares: two locus model; circles: three locus model; and diamonds: four locus model. Small sized symbols are for bottleneck of size 2, medium sized symbols are for bottlenecks of size 4, and large sized symbols are for bottlenecks of size 8 .

\section{Discussion}

Before discussing our results, a few words are necessary concerning the model and the metric we used. The effect of bottlenecks on genetic variance could be modeled using either of two approaches. One is the coancestry approach, first described by Goodnight (1988), which has the advantage of being independent of allelic frequencies, but requires trickier mathematics. The other is the Markov Chain model we used. One drawback of this method is that it depends on allele frequencies, even if Goodnight (2000) showed that the Markov-Chain method gives results similar to the coancestry approach. To correct for a potential bias in the results due to starting allelic frequencies, we present the results of the model for a total of $11^{4}=14,641$ starting points from which drift is allowed to operate. We also choose a metric to quantify the release in additive variance (equation 7) that should be insensitive to initial levels of additive variance. Previous authors used the ratio of post- and prebottleneck additive variance to quantify the conversion (Willis and Orr 1993; LópezFanjul et al. 2002). This ratio suffers from being very sensitive to low levels of additive variance before the bottleneck (see for instance Fig. 3 of López-Fanjul et al. 2002). Here, we take the difference between post- and prebottleneck additive variance, a quantity less dependant on the initial additive variance. This difference is divided by the total prebottleneck total genetic variance (expected to be larger in models with four genes than in model with two, see Keightley 1989) to make amount of additive variance comparable between the two, three and four locus models. 
As gene number increases, the number of possible epistatic interactions increases exponentially. We show here that this increase in gene number translates in an increase in the proportion of epistatic variance present before a bottleneck (Table 1). We also show that after a population bottleneck, the release in additive variance increases with the number of genes for all the matrices of genotypic values studied except the Dominant Metabolic Pathway (DMP). The two models (AA and AMP) with neither dominance nor dominance interactions (and therefore for which a transfer of variance from dominance to additivity cannot be suspected) are also the models where the release in additive variance is maximum. AA epistasis (the only form of epistasis present in the AA and AMP models) is the only form of epistasis substantially converted in additive variance. This is so even though the initial proportion of epistatic variance is lower for "pure" AA than for AD and DD epistasis (Table 1). For additive variance to increase, the type of epistasis is therefore more prominent than the initial fraction of the genetic variance it represents. This was already noticed for two loci by Goodnight (1988), Cheverud and Routman (1996) and Cheverud (2000), but our results generalize this conclusion.

\section{Respective Roles of Dominance and Epistasis}

It is common knowledge that when only additive variance is present, the effect of a bottleneck is to decrease it by a factor (1-F) (see Lande 1980; Lynch and Walsh 1998). However, in a two locus model restricted to AA epistasis, Goodnight (1988) showed that the additive variance is enhanced after population bottlenecks whenever $\sigma_{A A}^{2} / \sigma_{A}^{2}>1 / 3$, because the loss in additive variance due to inbreeding is balanced by the transfer from epistasis to additive variance. Our results show that this prediction seems to hold for two, three, and four genes. Indeed, for the AA model, $\sigma_{A A}^{2} / \sigma_{A}^{2}$ is always higher than 0.43 (Table 1) and an increase in additive variance is observed (Fig. 1). For the AMP model with two genes, $\sigma_{A A}^{2} /$ $\sigma_{A}^{2}$ is less than one-third $(0.19$, Table 1$)$ and the additive variance always decreases (Fig. 3), whereas for three and four genes, $\sigma_{A A}^{2} / \sigma_{A}^{2}$ is larger or equal to one-third, and an increase in additive variance is observed. This increase in additive variance even when $\sigma_{A A}^{2} / \sigma_{A}^{2}=1 / 3$ for the three locus model is an indication that the conditions for this increase for three genes are perhaps less strict than for two genes. This is perhaps due to the value of $\sigma_{A A}^{2}$ not being necessarily representative of (and generally underestimating) the amount of individual-level epistasis.

Other types of epistasis have a less pronounced effect on the release in additive variance as exemplified by DD "pure" epistasis and to a lesser extend by AD "pure" epistasis (Fig. 1 ), even though the initial proportion of total epistatic variance is higher for both $\mathrm{AD}$ and DD than for AA epistasis (Table 1). It remains that even when epistasis is not purely additive-by-additive (our method of estimation of the different variance components does not allow to distinguish the different epistatic terms, see above), with three and four loci, we still see an increase in the proportion of additive variance following a bottleneck (Figs. 1 and 2).

From our results, dominance variance seems to have a very limited influence on the release in additive variance after population bottlenecks. First, the initial proportion of dominance variance decreases with the number of genes (Table 1) for all the matrices of genotypic values explored. Thus, it is difficult to explain the increased release in additive variance with the number of loci by the sole transfer from dominance to additivity. Robertson (1952), Rose (1982), and Willis and Orr (1993) have shown that dominance variance can be converted into additive variance and could therefore counterbalance the loss in additive variance with inbreeding. This is in particular the case when genetic drift increases the frequency of a rare recessive allele. But averaged over the allele frequency space, a pure dominance model with one locus (a $=0, \mathrm{~d}=1$ ) leads to an average decrease in additive variance following a bottleneck (for instance, the decrease in additive variance after a bottleneck of two individuals averaged over initial frequencies $0.1,0.2 \ldots 0.9$ is $18 \%$ ). This comes from two separate processes. First, the additive variance itself decreases. Second, dominance variance is converted into additive variance for a large proportion of the allele frequency spectrum, but this is not sufficient to balance the loss of $\sigma_{A}^{2}$ over the whole frequency space.

The DMP model (Fig. 3) with four genes is particularly informative on this issue. In this model, a strong decrease in additive variance ( $48 \%$ on average initially, Table 1 ) is observed despite the presence of dominance (14\%) and epistatic $(38 \%)$ variance in the initial infinite population. A near linear negative relation between $R_{\left(t_{i}-t_{0}\right)}$ and $F$ is seen for two, three, and four loci. This decrease is the pattern expected under a pure additive model. The results for the AMP model are quite different. In this model (with $52 \% \sigma_{\AA}^{2}$, an initial proportion similar to the DMP model, and $48 \% \sigma_{1}^{2}$, Table 1) additive variance increases quite dramatically after the successive bottlenecks (Fig. 3). What differs between these two models are first that there is no dominance variance in the AMP model, and second, that the epistatic variance in the AMP model is exclusively of the AA type (There can be no AD or DD epistatic variance with no dominance). In these two models at least, it is clear that dominance variance is not the main factor in the transfer to additive variance following bottlenecks, but that AA epistatic variance plays a prominent role. Because AA epistasis does not contribute to inbreeding depression (see Lynch and Walsh 1998, p. 258), this increase in additive variance will not be penalized by inbreeding depression, in opposition to what was suggested by LópezFanjul et al. (2002). A further difference between our findings and those of López-Fanjul et al. (2002) is that we found conversion occurring despite initial additive variance being present.

\section{Respective Roles of Bottleneck Size and Duration}

Different levels of inbreeding were obtained by changing both the size and number of successive bottlenecks. Previous authors (e.g., Cheverud and Routman 1995) emphasized the effect of bottleneck size on the rate of conversion of epistatic variance. However, Walsh and Lynch (1998) have suggested that the important factor is neither the size of the bottleneck nor its duration, but rather the inbreeding level attained by the population. We confirm here that maximum releases in additive variance were obtained for given inbreeding levels 
independently of the bottleneck size and we also show that increasing the number and level of interactions between loci increases the level of inbreeding after which a decline in additive variance is observed after population bottlenecks.

\section{How Many Loci Are Concerned in Nature?}

The present study was limited to the simulation of four different independent genes displaying epistasis. This number of loci is more than what is found in theoretical investigations published to date, but still questionable because it is commonly agreed that quantitative traits are made of a large number of genes. Estimating the number of genes underlying variation has been the challenge of many geneticists, and has led to the development of different tools from the Castle-Wright estimator (Castle 1921; Wright 1968; Otto and Jones 2000) to QTL analysis (Lynch and Walsh 1998). According to the literature, the number of genes we simulated is in agreement with what is found in the literature for QTL analyses. As a matter of fact, QTL analyses usually identify between two and eight different QTLs with a mean of 4.21 and a standard deviation of 1.82 (reviewed in Roff 1997). However, other studies have pointed out that QTL analyses systematically underestimate the number of actual QTLs, either because of experimental designs with low power (Beavis 1994) or because QTLs of low average effects are structurally not detectable. Indeed, only QTLs with large or moderate effects are detected (Otto and Jones 2000). This indicates that the number of genes used in this study could be representative of a large number of characters whose determinism is directed by a small number of genes with large effects and a much higher number of genes with small effects. Because we have demonstrated that the release in additive variance after population bottlenecks increases with the number of genes, therefore we can expect that the observed pattern would be reinforced with traits directed by more than four genes, because epistatic interactions grow exponentially with the number of genes.

\section{Are There Epistatic Interactions in Nature?}

From our results, it is clear that epistasis, and in particular AA epistasis, plays a more prominent role than dominance in inflating the additive variance after population bottlenecks. However, the role that epistasis can play may only be significant if gene interactions are demonstrated to be common. This is what has been postulated in several models of evolutionary change, beside Wright's shifting balance theory, like Templeton's genetic transilience mode of speciation (Templeton 1980a,b; Carson 1982), or Schmalhausen's (1949) concept of stabilizing selection. Although Wright's principle of "universality of gene interactions" has been acknowledged by many geneticists, the occurrence of epistasis is still considerably discussed in both plants and animals. This is due to several factors, among them is the difficulty to measure gene interactions. There can indeed be substantial epistatic gene action (physiological and/or "pure", epistasis) but still little manifestation of this in the form of epistatic genetic variance within a population (statistical epistasis) (Cheverud and Routman 1995; Whitlock et al. 1995). For instance, Keightley (1989) showed that, when modeling epistasis in metabolic pathways, the epistatic variance is expected to be small in spite of the prevalence of epistatic interactions. The analysis of variance techniques, and the underlying Fisherian model of quantitative genetics classically used for analyzing variation, are also known to minimize epistatic variance since the least-mean-square analysis implicitly maximizes the main effects against interaction effects (see Templeton 2000; and Wade 1992, p. 44, and his description of Neyman's Monte Carlo experiment). Moreover, confidence limits for epistatic variance are generally larger than for the other fractions of the genetic variance, because of the small coefficient associated with the epistatic variance in the covariance among relatives. Also, focusing on the detection of interactions between two loci or QTLs might hide epistasis between three or more loci (Templeton 2000). These factors considerably increase the size of experimental designs needed to measure epistasis and therefore reduce the number of cases where designs are powerful enough to detect gene interactions. Nevertheless, it seems that epistasis is often found when adequate designs are used. This is particularly the case with crop plants for which extensive analyses have been conducted for improvement purposes (among others, Bailey et al. (1980) on wheat; Vasquez and Sanchez-Monge (1987) and Choo and Reinberg (1988) on barley; Moreno-Gonzalez and Dudley (1981), Damerval et al. (1994), Doebley et al. (1995) on maize; Lark et al. (1995) on soybean; Li et al. (1997) on rice, and MirzaieNodoushan et al. (1999) on red clover), or for some laboratory models like Drosophila (Long et al. 1995; Clark and Wang 1997) or mice (Cheverud and Routman 1996). Epistasis has also been found extensively in humans (for review, see Templeton 2000).

QTL identification and analysis are a promising method in this field, because they allow to measure epistatic effects rather than epistatic variance (Kao et al. 1999; Templeton 2000; Jannink and Jansen 2001; Kao and Zeng 2002) as exemplified by Cheverud and Routman (1996), Eshed and Zamir (1996), Cockerham and Zeng (1996), Bailey et al. (1997), Routman and Cheverud (1997), Gurganus et al. (1999), Kao et al. (1999), Shook and Johnson (1999), and Ungerer et al. (2002), among others. This is an important outcome since the evolutionary potential of allelic combinations depends on their epistatic effects, rather than their epistatic variance (Whitlock et al. 1995).

\section{How Applicable Are These Results to Traits under Selection?}

We did not include the effect of selection (nor did all other theoretical studies of this kind) because our main goal was to estimate the effect of the number of genes on the release in additive variance after population bottlenecks. Beside the intrinsic interest of quantifying the effect of a bottleneck on neutral genetic variance, focalizing on neutral genes is also tenable for two reasons. First, when a population is submitted to a bottleneck, drift should become the prominent force affecting allele frequencies changes, even if selection of moderate strength is present. Second, one expects the additive variance for a selected trait to be small in a population at equilibrium, while the other component of genetic variance 
can be quite important (Mousseau and Roff 1987; Roff and Mousseau 1987). Because the proportion of additive variance for a selected trait before a bottleneck is expected to be close to zero, changes in allele frequencies due to a bottleneck are therefore bound to increase the amount of additive variance by epistasis and/or dominance conversion, and allele frequencies at equilibrium are likely those from which one expects the largest amount of conversion. As the increase in additive variance reported here is an average taken over all the surface of possible allele frequencies, and therefore frequencies for which $\sigma_{A}^{2} \neq 0$, it is likely that our results are an underestimate of what is expected from selected traits at equilibrium (see López-Fanjul et al. 2002).

The former arguments, while explaining the adequacy of a neutral model in the context of small populations, clearly do not dismiss the need for more complex and realistic models including selection. It remains that our results show that epistasis, rather than dominance, plays a significant role in the increase of additive variance following bottlenecks.

\section{ACKNOWLEDGMENTS}

This work was supported by the Swiss National Science Foundation, grant no. 32-55036.98 to YN-G. and grants no. 31-05945.98, 31-59326.99, and 31-68325.02 to JG. YN-G was also supported by the two following foundations: Fondation du 450 ème Anniversaire de l'Université de Lausanne and Association Genevoise des Femmes Diplômées des Universités.

\section{Literature Cited}

Bailey, T. B., C. O. Qualset, Jr., and D. F. Cox. 1980. Predicting heterosis in wheat. Crop Sci. 20:339-342.

Bailey, M. A., M. A. R. Mian, T. E. Carter, D. A. Ashley, and H. R. Boerma. 1997. Pod dehiscence of soybean: identification of quantitative trait loci. J. Hered. 88:152-154.

Beavis, W. D. 1994. The power and deceit of QTL experiments: lessons from comparative QTL studies. Pp. 252-268 in the 49th Annual corn and sorghum research conference. American Seed Trade Assn., Washington, DC.

Bost, B., C. Dillmann, and D. de Vienne. 1999. Fluxes and metabolic pools as model traits for quantitative genetics. I. The Lshaped distribution of genes. Genetics 153:2001-2012.

Bryant, E. H., and L. M. Meffert. 1995. An analysis of selectional response in relation to a population bottleneck. Evolution 49: 626-634.

Bryant, E. H., and L. M. Meffert. 1996. Nonadditive genetic structuring of morphometric variation in relation to a population bottleneck. Heredity 77:168-176.

Carson, H. L. 1982. Speciation as a major reorganization of polygenic balance. Pp. 411-433 in C. Barigozzi, ed. Mechanisms of speciation. Alan Liss, New York.

Castle, W. E. 1921. An improved method of estimating the number of genetic factors concerned in case of blending inheritance. Proc. Natl. Acad. Sci. USA 81:6904-6907.

Cheverud, J. M. 2000. Detecting epistasis among quantitative trait loci. Pp. 58-81 in J. B. Wolf, E. D. Broodie III, and M. J. Wade, eds. Epistasis and the evolutionary process. Oxford Univ. Press, New York.

Cheverud, J. M., and E. J. Routman. 1995. Epistasis and its contribution to genetic variance components. Genetics 139: $1455-1461$.

1996. Epistasis as a source of increased additive genetic variance at population bottlenecks. Evolution 50:1042-1051.

Cheverud, J. M., T. T. Vaughn, L. S. Pletscher, K. King-Ellison, J. Bailiff, E. Adams, C. Erickson, and A. Bonislawski. 1999
Epistasis and the evolution of additive genetic variance in populations that pass through a bottleneck. Evolution 53:1009-1018.

Choo, T. M., and E. Reinberg. 1988. Diallel analysis of heading date in barley using doubled haploid lines. Genet. Agrar. 42: 202-212.

Clark, A. G., and L. Wang. 1997. Epistasis in measured genotypes: drosophila P-element insertions. Genetics 147:157-163.

Cockerham, C., and H. Tashida. 1988. Permanency of response to selection for quantitative characters in finite populations. Proc. Natl. Acad. Sci. USA 84:6205-6209.

Cockerham, C., and Z. B. Zeng. 1996. Design III with marker loci. Genetics 143:1437-1456.

Damerval, C., A. Maurice, J. M. Josse, and D. de Vienne. 1994 Quantitative trait loci underlying gene product variation: a novel perspective by analyzing regulation of genome expression. Genetics 137:289-301.

Doebley, J., A. Stec and C. Gustus. 1995. theosinthe branchedl and the origin of maize: evidence for epistasis and the evolution of dominance. Genetics 141:333-346.

Eshed, Y., and D. Zamir. 1996. Less-then-additive interactions of quantitative trait loci in tomato. Genetics 143:1807-1817.

Falconer, D. S., and T. Mackay. 1996. Introduction to quantitative genetics, 4th ed. Longman, Harlow, U.K.

Fernández, A., M. A. Toro, and C. López-Fanjul. 1995. The effect of inbreeding on the redistribution of genetic variance of fecundity and viability in Tribolium castaneum. Heredity 75: 376-381.

Fischer, R. A. 1958. The genetical theory of natural selection. 2d ed. Dover, New York.

García, N., C. López-Fanjul, and A. García-Dorado. 1994. The genetics of viability in Drosophila melanogaster: effects of inbreeding and artificial selection. Evolution 48:1277-1285.

Goodnight, C. J. 1987. On the effect of founder events on the additive genetic variance. Evolution 41:171-179.

1988. Epistasis and the effect of founder events on the additive genetic variance. Evolution 42:441-454.

2000. Modeling gene interaction in structured populations. Pp. 129-145 in J. B. Wolf, E. D. Broodie III, and M. J. Wade, eds. Epistasis and the evolutionary process. Oxford Univ. Press, New York.

Gurganus, M. C., S. V. Nuzhdin, J. W. Leips, and T. F. C. Mackay. 1999. High-resolution mapping of quantitative trait loci for sternopleural bristle number in Drosophila melanogaster. Genetics 152:1585-1604.

Hartl, D. L., and A. G. Clark. 1997. Principle of population genetics, 3rd ed. Sinauer Associates, Sunderland, MA.

Jannink, J. L., and R. Jansen 2001. Mapping epistatic quantitative trait loci with one dimentional genome search. Genetics 157: $445-454$.

Kao, C. H., and Z. B. Zeng. 2002. Modeling epistasis of quantitative trait loci using Cockerham's model. Genetics 160:1243-1261.

Kao, C. H., Z. B. Zeng, and R. D. Taesdale, 1999. Multiple interval mapping for quantitative trait loci. Genetics 152:1203-1216.

Keightley, P. R. 1989. Models of quantitative variation of flux in metabolic pathways. Genetics 121:869-876.

Keightley, P. R., and A. Eyre-Walker. 1999. Terumi Mukai and the riddle of deleterious mutation rates. Genetics, 153:515-523.

Lande, R. 1980. Genetic variation and phenotypic evolution during allopatric speciation. Am. Nat. 116:463-479.

Lark, K. G., K. Chase, F. Alder, L. M. Mansur, and J. F. Orf. 1995. Interactions between quantitative trait loci in soybean in which trait variation at one locus is conditional upon a specific allele at another. Proc. Natl. Acad. Sci. USA 92:4656-4660.

Li, Z., S. R. Pinson, W. D. Park, A. H. Paterson, and J. W. Stansel. 1997. Epistasis for three grain yield components in rice (Oryza sativa L.). Genetics 145:453-465.

Long, A. D., S. L. Mullaney, L. A. Reid, J. D. Fry, C. H. Langley, and T. F. C. Mackay. 1995. High resolution mapping of genetic factors affecting abdominal bristle number in Drosophila melanogaster. Genetics 139:1273-1291.

López-Fanjul, C., and A. Villaverde. 1989. Inbreeding increases genetic variance for viability in Drosophila melanogaster. Evolution 43:1800-1804. 
López-Fanjul, C., A. Fernandez, and M. A. Toro. 1999. The role of epistasis in the increase in the additive genetic variance after population bottlenecks. Genet. Res. Camb. 73:45-59.

2000. Epistasis and the conversion of non-additive to additive genetic variance at population bottlenecks. Theor. Popul. Biol. 58:49-59.

- 2002. The effect of epistasis on the excess of the additive and nonadditive variances after population bottlenecks. Evolution. 56:865-876.

Lynch, M., and B. Walsh. 1998. Genetics and analysis of quantitative traits. Sinauer Associates, Sunderland, MA.

Lynch, M., J. Conery, and R. Bürger. 1995. Mutational meltdown in sexual populations. Evolution 49:1067-1080.

Meffert, L. 2000. The evolutionary potential of morphology and mating behavior: The role of epistasis in bottlenecked populations. Pp. 177-193 in J. B. Wolf, E. D. Broodie III, and M. J. Wade, eds. Epistasis and the evolutionary process. Oxford Univ. Press, New York.

Merilä, J., and B. C. Sheldon. 1999. Genetic architecture of fitness and nonfitness traits: empirical patterns and development of ideas. Heredity 83:109-109.

Mirzaie-Nodoushan, H., I. L. Gordon, and W. B. Rumball, 1999. Inheritance of growth habit-related attributes in red clover (Trifolium pratense L.). J. Hered., 90:550-553.

Moreno-Gonzalez, J., and J. W. Dudley. 1981. Epistasis in related and unrelated maize hybrids determined by three methods. Crop Sci. 21:644-651.

Mousseau, T. A., and D. A. Roff. 1987. Natural selection and heritability of fitness components. Heredity 59:181-197.

Nei, M., T. Maruyama, and R. Chackraborty. 1975. The bottleneck effect and genetic variability in populations. Evolution 29:1-10.

Otto, S. P., and C. D. Jones. 2000. Detecting the undetected: estimating the total number of loci inderlying a quantitative trait. Genetics 156:2093-2107.

Robertson, A. 1952. The effect of inbreeding on the variation due to recessive genes. Genetics 37:189-207.

Roff, D. A. 1997. Evolutionary Quantitative Genetics. Chapman and Hall, New York.

Roff, D. A., and T. A. Mousseau. 1987. Quantitative genetics and fitness: lessons from Drosophila. Heredity 59:103-118.

Rose, M. 1982. Antagonistic pleiotropy, dominance and genetic variation. Heredity 48:63-78.

Routman, E. J., and J. M. Cheverud. 1997. Gene effects on a quantitative trait: two-locus epistatic effects measured at microsatellite markers and at estimated QTL. Evolution 51:1654-1662.

Saccheri, I. J., P. M. Brakefield, and R. A. Nichols. 1996. Severe inbreeding depression and rapid fitness rebound in the butterfly Bicyclus anynana (Satyridae). Evolution 50:2000-2013.

Schmalhausen, I. 1949. Factors of evolution. Blackiston, Philadelphia, PA.
Shook, D. R., and T. E. Jonhson, 1999. Quantitative trait loci affecting survival and fertility-related traits in Caenorhabditis elegans show genotype-environment interactions, pleiotropy, and epistasis. Genetics 153:1233-1243.

Templeton, A. 1980a. The theory of speciation via the founder principle. Genetics 94:1011-1038.

. 1980b. Mode of speciation and interference based on genetic distances. Evolution 34:719-729.

. 2000. Epistasis and complex traits. Pp 41-57 in J. B. Wolf, E. D. Broodie III, and M. J. Wade, eds. Epistasis and the evolutionary process. Oxford Univ. Press, New York.

Ungerer, M. C., S. S. Halldorsdottir, J. L. Modliszewski, T. F. C. Mackay, and M. D. Purugganan. 2002. Quantitative trait Loci for inflorescence development in Arabidopsis thaliana. Genetics, 160:1133-1151.

Vasquez, J. F., and E. Sanchez-Monge. 1987. Correlation, epistasis and heterosis of plant height and internode length in barley. Genome 29:532-536.

Wade, M. J. 1992. Sewall Wright: gene interaction in the shifting balance theory. Pp. 35-62 in J. Antonovics and D. Futuyma, eds. Oxford surveys of evolutionary biology. VI. Oxford Univ. Press, New York.

Wade, M. J., S. M. Shuster, and L. Stevens. 1996. Inbreeding: its effects on response to selection for pupal weight and the heritable variance in fitness in the flour beetle, Tribolium castaneum. Evolution 50:723-733.

Walsh, B., and M. Lynch, 1998. Evolution and selection of quantitative traits. http://nitro.biosci.arizona.edu/zbook/volume_2/ vol2.html.

Wang, J., A. Caballero, P. D. Keightley, and W. G. Hill. 1998. Bottleneck effect on genetic variance: a theoretical investigation of the role of dominance. Genetics 150:435-447.

Willis, J., and H. A. Orr. 1993. Increased heritable variation following population bottleneck: the role of dominance. Evolution 47:949-957.

Whitlock, M. C., P. C. Phillips, and M. J. Wade. 1993. Gene interaction affects the additive genetic variance in subdivided populations with migration and extinction. Evolution 47:1758-1769.

Whitlock, M. C., P. C. Phillips, F. B. G. Moore, and S. J. Tonsor. 1995. Multiple fitness peaks and epistasis. Annu. Rev. Ecol. Syst. 26:601-629.

Wolf, J. B., E. D. Broodie III, and M. J. Wade. 2000. Epistasis and the evolutionary process. Oxford Univ. Press. New York.

Wright, S. 1931. Evolution in Mendelian populations. Genetics 16: 97-159.

- 1968. Evolution and the genetics of populations. Vol 1. Genetic and biometric fondations. Univ. of Chicago Press, Chicago, IL.

Corresponding Editor: J. Cheverud 
APPENDIX

Genotypic values for "pure" form of epistasis (AA, AD, and DD) and for physiological epistasis (additive and dominant metabolic pathways).

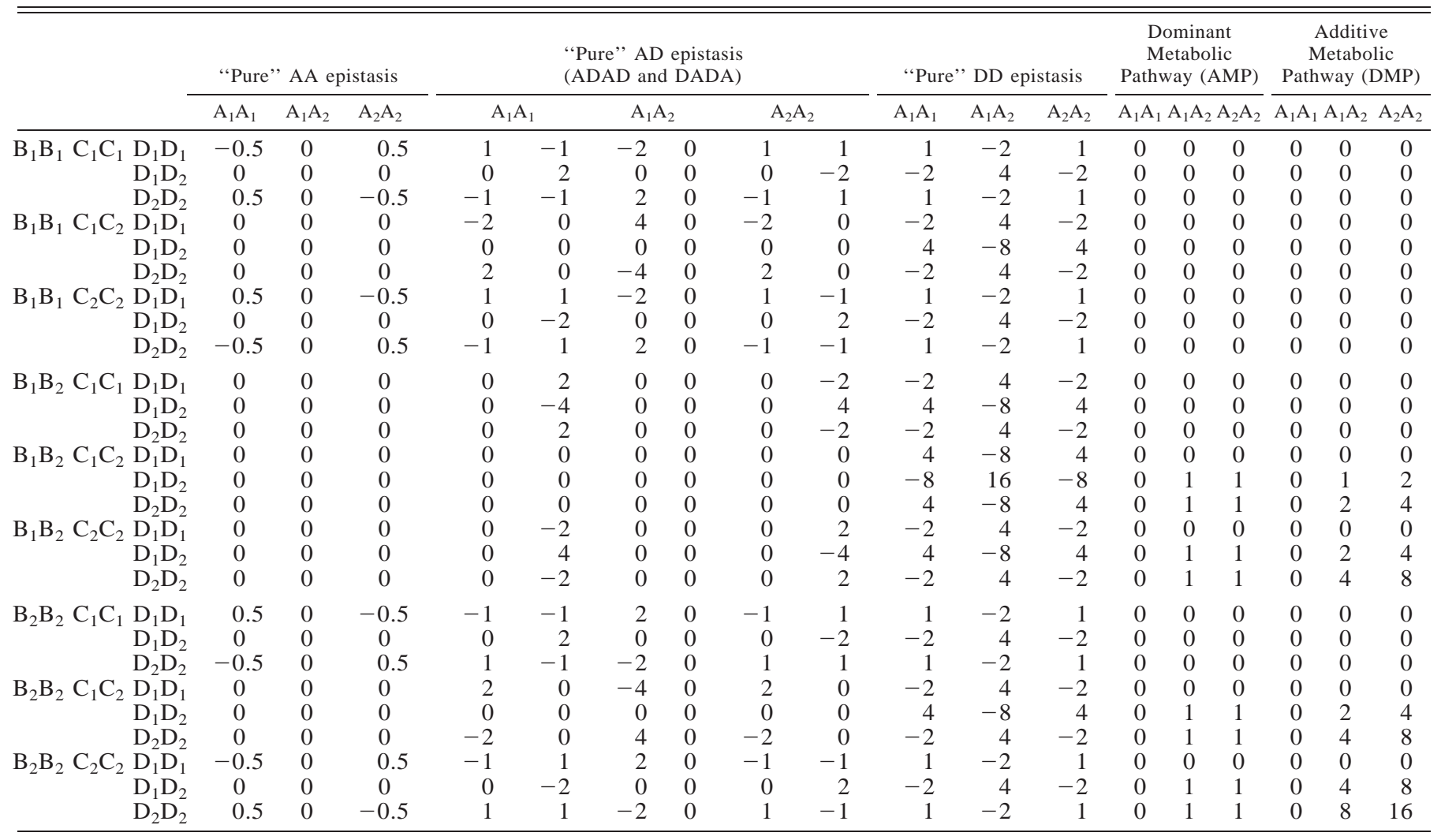

